low acuity issues that could potentially be avoided with counseling or supplies at the initial visit. However, higher acuity re-registrations, both within and between event years, are a higher risk for transport and could benefit from early identification. Having a plan to identify and potentially remove the sicker, higher risk patients from the event could be important for safety and liability.

Prehosp Disaster Med 2019;34(Suppl. 1):s178-s179

doi:10.1017/S1049023X19004102

Why Patients Refuse to Enroll in Hospital-Based Research: Perception of Patients Presenting to KATH Emergency Department

Dr. Joseph Bonney, Ms. Esi Amissah, Ms. Sonia Cobbold,

Dr. Paa Kobina Forson

Emergency Medicine, Komfo Anokye Teaching Hospital, Kumasi, Ghana

Introduction: Komfo Anokye Teaching Hospital Emergency Department (KATH ED) is a tertiary referral center in Ghana. Anecdotally, patients seeking care at KATH ED do not actively participate in research initiatives.

Aim: To find out why patients presenting to KATH ED do not enroll in research studies that are conducted in the department. Methods: The study was a cross-sectional survey of patients presenting to the ED for one month in June 2017. A semistructured questionnaire was presented to patients presenting to KATH ED from 8:00-20:00 each day. Patients who were interviewed were all patients presenting to the ED for care, including those who had refused to enroll in the ongoing ACESO study. Patients had to be conscious, alert, and with conditions that did not require immediate management.

Results: $35 \%$ of the interviewees (91/260) had been approached to enroll in research studies at some point in the past. $13.5 \%$ had refused to enroll in a research study. $45.7 \%$ of those who refused to enroll admitted that they were afraid to enroll in a study; $28.6 \%$ had inadequate information and $22.9 \%$ perceived enrolling in a study would delay their treatment. The Akan language (73\%) was most commonly used by research assistants then English (26\%), and finally Hausa (1\%) to interact with patients. There was a significant association between educational background and explaining a study to a patient before they enrolled. Males were more willing to enroll in an ongoing study compared to females. All age groups correlated significantly with being approached to enroll in a research study and similarly all age groups also correlated with refusing to enroll in a research study

Discussion: Patients are paramount to hospital research. Efforts must be made to ensure that patients concerns and needs are addressed to ensure increasing participation.

Prehosp Disaster Med 2019;34(Suppl. 1):s179

doi:10.1017/S1049023X19004114

Working Together to Develop Best Practice: Rescue Operations in Confined Settings

Ms. Sofia Karlsson, Dr. Lina Gyllencreutz,

Prof. Britt-Inger Saveman
Department of Surgical and Perioperative Sciences, Centre for Research and Development in Disaster Medicine, Umeå University, Umeå, Sweden

Introduction: Major injury incidents in confined settings such as tunnels and underground mineral- and metalliferous mines are rare, but when they do happen, the consequences may be severe with potential for many injured. The incident site is underground and it is difficult for the rescue and emergency medical service to get an overview and reach the injured. Therefore, it is important for the emergency medical service, rescue service, and the company responsible for the underground environment to have a good collaboration.

Aim: To develop best practices of conducting rescue response from a disaster medicine perspective in tunnels and underground mines through increased education.

Method: Within an EU-program, the university collaborates with stakeholders such as rescue service, emergency medical service, and two mining companies. Within this project, an explorative case study with participatory research is conducted. This is managed with the help of representatives of the stakeholders, workshops, and through planning for and conducting observations of table-top and full-scale exercises.

Results: At the first workshop the stakeholders built a timeline presenting their activities from a major incident occurring in an underground mine until the last injured was transported to the hospital. Thereafter, several workshops were conducted to find improvements that could be made regarding collaboration between the organizations. Table-top and full-scale exercises have also revealed further challenges. Within the project, prototypes are being developed and will be presented during the conference.

Discussion: This project involves stakeholders in the research process, and they, therefore, have a direct impact on the development of best practices of rescue in major underground incidents.

Prehosp Disaster Med 2019;34(Suppl. 1):s179

doi:10.1017/S1049023X19004126

\section{A Workplace-Based Observation Strategy to Assess Prehospital Care Delivery by Public Ambulances in Ukraine Mr. Stanislav Gaierskyi ${ }^{1}$, Dr. Oleksandr Lincherskyy ${ }^{2}$, Dr. Colin Meghoo ${ }^{3}$ \\ 1. Kyiv-Mohyla Academy, Kiev, Ukraine \\ 2. Ministry of Health, Kiev, Ukraine \\ 3. Patriot Defence, Kiev, Ukraine}

Introduction: Current methods to evaluate the delivery of urgent prehospital care often rely on inadequate surrogate measures or unreliable self-reported data. A workplace-based strategy may be feasible to assess the delivery of prehospital care by ambulances in selected populations.

Aim: To perform a nationwide assessment of the psychomotor performance of public ambulance workers in Ukraine, we created a plan of workplace-based observation. We conducted a post-hoc analysis of this strategy to assess feasibility, strengths, 